\title{
Representación del \\ significado léxico de \\ tarea escolar: la visión \\ de los estudiantes*
}

\author{
Representation \\ of the Lexical \\ Meaning of \\ Homework: the \\ Students' Vision
}

\author{
Representação \\ do significado \\ lexical da dever \\ escolar: a visão \\ dos alunos
}

Jaime Ruiz Vega** Orcid: https://orcid.org/0000-0001-6116-8876

Álvaro William Santiago Galvis*3 Orcid: https://orcid.org/000-0002-2137-0137

Myriam Cecilia Castillo Perilla*4 Orcid: https://orcid.org/0000-0002-3452-5003

* Los planteamientos que se exponen a lo largo de este documento surgen de la ejecución del proyecto de investigación Concepciones de los estudiantes de educación básica secundaria y media de Bogotá sobre la entidad léxica tarea escolar (DLE-427-16), financiado por el Centro de Investigaciones de la Universidad Pedagógica Nacional (ciup). Asimismo, complementan los datos presentados en el artículo La tarea escolar: representaciones lingüísticas de los estudiantes (Ruiz, Santiago y Castillo, 2018).

** Q.E.P.D. Doctor en Ciencias del Lenguaje de la Universidad de Nantes, Francia. Docente del Departamento de Lenguas de la Universidad Pedagógica Nacional, integrante del Grupo de Investigación en Pedagogía, Lenguaje , Universidad Pedagógica Nacionaly Comunicación, GIPELEC.

Correo electrónico: jruiz@pedagogica.edu.co

*3 Magister en Lingüística Hispánica del Instituto Caro y Cuervo, Bogotá, Colombia. Docente del Departamento de Lenguas de la Universidad Pedagógica Nacional, integrante del Grupo de Investigación en Pedagogía, Lenguaje y Comunicación, GIPELEC.

Correo electrónico: asantiago@pedagogica.edu.co

*4 Magister en Literatura Hispanoamericana del Instituto Caro y Cuervo, Bogotá, Colombia. Docente del Departamento de Lenguas de la Universidad Pedagógica Nacional, integrante del Grupo de Investigación en Pedagogía, Lenguaje y Comunicación, GIPELEC.

Correo electrónico: mcastillo@pedagogica.edu.co.

\section{Para citar este artículo}

Ruiz, J. y Santiago, W. y Castillo, M. (2020). Representación del significado léxico de tarea escolar: la visión de los estudiantes. Folios, 51, 79-97.

doi:10.17227/folios.51-8644<smiles>[C]=C</smiles>

Artículo recibido $01 \cdot 11 \cdot 2018$

Artículo aprobado $25 \cdot 07 \cdot 2019$ 


\title{
Resumen
}

Este artículo presenta otra parte de los resultados del proyecto de investigación Concepciones de los estudiantes de educación básica secundaria y media de Bogotá sobre la entidad léxica tarea escolar (DLE-427-16), sustentado en los planteamientos de la Semántica de los Posibles Argumentativos (SPA); orientación teórica que centra su interés en el estudio del significado léxico y su relación con los valores, así como con las representaciones, individuales y sociales, que las formas léxicas vehiculan. Esto se sustenta en el planteamiento de que los hablantes de una lengua, en el uso discursivo, ofrecen una axiologización discursiva del mundo que se hace evidente en el nivel léxico. Para efectos de establecer dicha axiologización se recurre, metodológicamente, al análisis de corpus léxicos obtenidos a partir de la aplicación de cuestionarios que permiten tener una muestra de la producción discursiva de los hablantes, en este caso, estudiantes de educación básica secundaria y media de Bogotá. En este texto se ofrece el análisis de los datos obtenidos, fundamentalmente en lo que respecta a los encadenamientos argumentativos derivados del significado léxico, así como de los discursos de los estudiantes que reclaman la continuidad de las tareas como una actividad escolar necesaria y útil; de esta forma, el texto ofrece las razones que la población en cuestión esgrimió para que la tarea escolar se mantenga como parte del proceso de formación de los individuos.

\author{
Palabras clave \\ tarea escolar; representación mental; léxico; estereotipo; semántica
}

\begin{abstract}
This article presents another part of the results of the research project Concepciones de los estudiantes de educación básica secundaria y media de Bogotá sobre la entidad léxica tarea escolar (DLE -427-16), based on the approaches of the Semantics of the Argumentative Possibilities (SPA); theoretical orientation that centers its interest in the study of the lexical meaning and its relation with the values, as well as with the representations, individual and social, that the lexical forms carry. This is based on the approach that speakers of a language, in discursive use, offer a discursive axiologization of the world that is evident at the lexical level. For the purpose of establishing this axiologization, is used, methodologically, the analysis of a lexical corpus obtained from the application of questionnaires that allow us to have a sample of the discursive production of speakers, in this case, secondary and middle school students in Bogotá. This text offers the analysis of the data obtained, mainly with regard to the argumentative chains derived from the lexical meaning, as well as the discourses of the students who demand the continuity of the homework as a necessary and useful school activity. In this way, the text offers the reasons that the population in question put forward so that the homework is maintained as part of the process of educating individuals.
\end{abstract}

\section{Keywords}

homework; mental representation; lexicon; stereotype; semantics

\section{Resumo}

Este artigo apresenta outra parte dos resultados do projeto de pesquisa Concepciones de los estudiantes de educación básica secundaria y media de Bogotá sobre la entidad léxica tarea escolar (DLE -427-16), com base nas abordagens da Semântica das Possibilidades Argumentativas (SPA); orientação teórica que centraliza seu interesse no estudo do significado lexical e sua relação com os valores, bem como com as representações individuais e sociais que as formas lexicais carregam. Isso se baseia na abordagem que os falantes de uma língua, em uso discursivo, oferecem uma axiologização discursiva do mundo que é evidente no nível lexical. Com o objetivo de estabelecer essa axiologização, a análise do corpus lexical obtido a partir da aplicação de questionários que permitem ter uma amostra da produção discursiva dos falantes é utilizada metodologicamente, neste caso, alunos do ensino médio e do ensino médio em Bogotá. Este texto oferece a análise dos dados obtidos, principalmente no que diz respeito às cadeias argumentativas derivadas do significado lexical, bem como os discursos dos alunos que demandam a continuidade das tarefas como atividade escolar necessária e útil. Dessa forma, o texto apresenta as razões apresentadas pela população em questão, para que os deveres escolares sejam mantidos como parte do processo de formação de indivíduos.

\section{Palavras-chave}

dever de casa; representação mental; léxico; estereótipo; semântica 


\section{Presentación}

En la misma línea del proyecto desarrollado con docentes del distrito capital, ${ }^{1}$ se indagó entre los otros actores fundamentales del proceso educativo, esto es, los estudiantes, con el objeto de determinar lo que discursivamente han construido a partir de las prácticas escolares, en particular, y de las acciones sociales, en general, en lo que respecta al tema de las tareas escolares. Solamente el estudio juicioso delas representaciones y de los valores portados por los discursos de estos sujetos puede arrojar elementos para comprender y enriquecer la problemática $y$, de esta manera, tomar decisiones en torno a esta actividad escolar sobre bases sólidas. Así, este proyecto tuvo como finalidad la identificación y el análisis de las concepciones de los estudiantes de educación básica secundaria y media de Bogotá sobre la entidad léxica tarea escolar; trabajo que se llevó a cabo bajo las orientaciones conceptuales y metodológicas de la Semántica de los Posibles Argumentativos (SPA).

\section{Consideraciones teóricas ${ }^{2}$}

\section{El significado léxico}

La SPA, como teoría semántica que busca dar cuenta de la inscripción de lo axiológico en el significado de las palabras, plantea que el significado de una forma léxica está configurado por cuatro estratos o niveles: el núcleo, los estereotipos, los posibles argumentativos y los despliegues argumentativos.

El núcleo "corresponde a los rasgos de categorización semántica descritos por Putnam (1975) como el elemento estable del significado léxico. La SPA considera estos rasgos como propiedades esenciales" (Ruiz, Castillo y Santiago, 2016, p.155). Los

1 Proyecto La movilización discursiva de valores sociales asociados a la entidad léxica tarea escolar en la educación básica y media en Bogotá, DLE-361-13, tuvo como objetivo analizar los valores sociales que de la entidad léxica tarea escolar tienen los docentes de lengua de los ciclos 1 a 5 de la ciudad de Bogotá (cfr. Castillo, Ruiz y Santiago, 2014; Ruiz, Castillo y Santiago, 2015; Ruiz, Castillo y Santiago, 2016).

2 Para tener un acercamiento más detallado a los planteamientos teóricos de la SPA, se puede revisar el trabajo de Castillo, Santiago y Ruiz, 2014 estereotipos se asumen como "un conjunto abierto de elementos del núcleo con otras representaciones y que constituyen bloques de argumentación interna" (Galatanu, 2009, p. 395). Los posibles argumentativos se originan en las relaciones que se establecen entre la entidad léxica y alguno de los estereotipos, de tal suerte que se constituyen en asociaciones virtuales, de argumentación externa, que "se organizan en dos haces orientados respectivamente hacia uno o el otro de los polos axiológicos (positivo y negativo)" (Galatanu, 2005, p. 57), lo cual hace que dicha relación esté de acuerdo o no con la orientación axiológica del estereotipo.

Los despliegues argumentativos, por su parte, corresponden a manifestaciones de orden discursivo, por lo cual se constituyen en la actualización o realización discursiva de los posibles argumentativos, esto es, "son las secuencias argumentativas realizadas por las ocurrencias discursivas" (Galatanu, 2009, p. 935). Este nivel se manifiesta en la acción discursiva, en la que se hacen evidentes los posibles argumentativos $\mathrm{y}$, a partir de éstos, es viable establecer la relación del sentido de las ocurrencias discursivas con el significado de la unidad léxica $y$, con base en ello, establecer la conformidad o no de dicho sentido con el protocolo semántico del significado de la unidad léxica en cuestión.

\section{La axiologización}

De acuerdo con lo planteado, la spa sostiene que en el uso de las formas léxicas, i. e., en la acción discursiva, el individuo ofrece una representación de su forma de ver y concebir la realidad factual, natural o sociocultural. Al respecto, Dervin afirma que "el análisis del discurso no se enfoca en saber si tal o cual enunciado es verídico, sino que se interesa ante todo en las representaciones que están presentes en el discurso" (2009, p. 170).

Según lo anterior, el discurso, en general, y las formas léxicas, en particular, llevan implícitos unos valores, de ahí que, desde la perspectiva de la SPA, el análisis del discurso supone el estudio de los valores $\mathrm{y}$, en consecuencia, se deben asumir los conceptos de modalización y de valor modal, de tal forma que "en 
el campo de la semántica, el modelo de la sPA busca mostrar que las entidades léxicas pueden tener uno o varios valores modales entre los elementos de sus estereotipos o incluso de sus núcleos" (Ruiz, Castillo y Santiago, 2016, p. 156).

Como se ha expuesto (cfr. Castillo, Santiago y Ruiz, 2014 y Ruiz, Castillo y Santiago, 2016, Ruiz, Santiago y Castillo, 2018), uno de los conceptos fundamentales para la SPA, desde la óptica de Galatanu (2000; 2002; 2003a; 2003b; 2007) corresponde al de valor modal, con el cual se hace referencia a la actitud del sujeto hablante con respecto a su enunciación o acción discursiva. Estas actitudes se agrupan en cuatro zonas semánticas modales: zona modal ontológica, zona modal de juicio de verdad, zona modal axiológica y zona modal de los valores finalizantes.

\section{Consideraciones metodológicas}

Con base en el protocolo que establece la SPA, el trabajo implicó la recolección, codificación, sistematización y análisis de las ocurrencias referidas a la entidad léxica tarea escolar. Para obtener dicho corpus se recurrió al cuestionario como instrumento para la recolección de la información. Este instrumento se diseñó para poder identificar y dar cuenta de los elementos del núcleo de la entidad léxica referida, los estereotipos y la orientación axiológica presente en las respuestas de los informantes. Para ello, el cuestionario se dividió en estos cuatro apartados: 1) pregunta abierta asociativa, para establecer los estereotipos; 2) pregunta cerrada excluyente, formulada para identificar la preferencia de los estudiantes en una propuesta de relaciones de los posibles argumentativos, esto gracias a la valoración que los informantes debían adelantar de una serie de encadenamientos argumentativos; 3) pregunta abierta en la que se solicitaba una definición de tarea escolar; y 4) pregunta abierta a través de la cual se requería una reacción con respecto a una situación hipotética, esto para tener un contraste de las representaciones y de los valores que los estudiantes movilizan en sus discursos (cfr. Ruiz, Santiago y Castillo, 2018).
En lo que respecta a la población, el trabajo se adelantó con un total de 712 estudiantes de educación básica y media, pertenecientes a instituciones educativas de carácter público de la ciudad de Bogotá, distribuidos así:

Tabla 1. Distribución de la población

\begin{tabular}{ccc} 
Ciclo & Grados & N. ${ }^{\circ}$ Estudiantes \\
\hline \multirow{2}{*}{3} & Sexto & 120 \\
\cline { 2 - 3 } & Séptimo & 127 \\
\hline \multirow{2}{*}{4} & Octavo & 121 \\
\cline { 2 - 3 } & Noveno & 119 \\
\hline \multirow{2}{*}{5} & Décimo & 89 \\
\cline { 2 - 3 } & Undécimo & 136
\end{tabular}

\section{Discusión de los resultados}

En este apartado se exponen el análisis y discusión de los datos que se obtuvieron de las respuestas que los estudiantes ofrecieron con respecto a las preguntas dos y cuatro del cuestionario. Como se planteó, en la segunda pregunta se le ofreció a la población una serie de siete encadenamientos argumentativos, con el fin de que determinara y estableciera si dichas relaciones eran posibles o no. En la cuarta pregunta, entre tanto, se les presentó una situación hipotética para que tomaran partido frente a ésta y sustentaran su decisión. De manera específica, en la segunda pregunta, al juzgar las cadenas argumentativas como posibles o imposibles, la población objeto de estudio revela las representaciones que tiene de los aspectos y las relaciones contemplados en cada una de ellas. Por lo tanto, la elección propuesta posible/ imposible permite determinar sus razonamientos y su concepción del mundo - de la tarea para el caso de esta investigación - que se estructura en sus experiencias vitales, escolares, en la influencia de discursos diversos. Por su parte, la cuarta pregunta favorece - como discurso motivado- la movilización de representaciones y de valores portados por éstas en torno a la tarea escolar. Las diversas justificaciones de la población interpelada contienen elementos del núcleo construido y reconstruido, así como de los estereotipos lexicográficos y de aquellos 
propuestos por tal población. De esta forma, en estas respuestas activan, refuerzan, neutralizan, debilitan e incluso invierten los valores sociales complejos que antes habían movilizado. Ello permite comparar y consolidar los primeros hallazgos (preguntas 1 y 3 ), por una parte, y confortar los análisis realizados (cf. Ruiz, Santiago y Castillo, 2018).

\section{Encadenamientos argumentativos}

Con base en el estudio del núcleo y de los estereotipos identificados en la construcción del significado léxico de tarea, a partir del discurso lexicográfico, se eligieron algunas entidades léxicas para elaborar cadenas argumentativas, cuyos elementos de enlace son los articuladores virtuales entonces (en adelante PLT) (conector normativo) y sin embargo (en adelante SE) (conector transgresivo) (cfr. Ruiz, Castillo y Santiago, 2016). Esto, con el fin de determinar las representaciones que los estudiantes tienen de tarea y la valoración que de esta actividad educativa reflejan en sus elecciones.

\section{Tarea entonces felicidad entonces recompensa}

El 67,79\% de estudiantes que acogieron esta posibilidad de encadenamiento introduce nuevas representaciones en el significado de tarea, ${ }^{3}$ lo cual no se encuentra acorde con el protocolo semántico construido a partir del discurso lexicográfico, en la medida en que ni en el núcleo de esta entidad léxica ni entre sus estereotipos se encuentran términos similares a felicidad o a recompensa (cfr. Ruiz, Santiago y, Castillo 2014). Se puede establecer, entonces, que estos componentes del significado no aparecen en el núcleo del significado lexicográfico, sino que se forjan

3 A partir de los diccionarios consultados (discurso lexicográfico, DRAE, DUE, Larousse, Educar, Norma) se estableció el núcleo de tarea escolar, así (Castillo, Santiago y Ruiz, 2014):

Tarea (escolar) N: Sustantivo $f$.

Obra y trabajo obligatorios PLT Dificultad plt Esfuerzo se Tiempo limitado PLT Resultado

Por otra parte, las respuestas que los estudiantes expusieron frente a la tercera pregunta del cuestionario permitieron reconstruir el significado léxico de la unidad lingüística tarea escolar, específicamente el núcleo, como sigue (Ruiz, Santiago y Castillo, 2018, p. 108):

Tarea (escolar) N: sustantivo f.

Trabajo y actividad obligatorios en casa PLT Entender y aprender tema PLT Nota. en el discurso de los informantes. Estas palabras probablemente se acuñan en las representaciones gracias a la influencia del discurso docente, de las interacciones escolares, de las acciones de los docentes y de los mismos padres de familia y/o acudientes. Así, las respuestas de los informantes permiten establecer que tienden a encontrar en la tarea que el profesor asigna un fin último de orientación pragmática: la nota (cfr. Ruiz, Santiago y Castillo, 2017), que es en cierto modo la equivalencia de recompensa que, a su vez, generaría felicidad.

Tarea entonces problema sin embargo mejoramiento

Un $83,88 \%$ de la población eligió esta cadena argumentativa como posible, lo que permite considerar que este subconjunto de estudiantes moviliza algunas entidades léxicas del significado de tarea, que las refuerza en sus representaciones al elegir este encadenamiento como posible. En este orden de ideas, los términos de esta cadena están de acuerdo con el protocolo semántico resultante del estudio lexicográfico y retoman un elemento de los estereotipos: ${ }^{4}$ problema. Este elemento se aproxima a dificultad, presente en el tercer bloque de significado del núcleo lexicográfico. De esta manera, el término problema retoma y precisa la idea presente en la forma léxica dificultad. Se trata de una reducción de las posibilidades contenidas en dificultad a especificidades ligadas a problema. El primer término supone un rango más amplio de representaciones, mientras que el segundo apunta a aspectos concretos del salón de clase, lo cual se justifica por las prácticas en las que los estudiantes están inmersos cotidianamente.

4 En la consulta de los diccionarios bases para la identificación de estereotipos de la unidad lingüística tarea escolar se estableció el siguiente conjunto de estereotipos: ejercicio (mental o físico), deber, ocupación, hacer en casa, lectura, escritura, proyecto, problema, práctica, construcción, conjunto de actividades, secuencia, estructuración, oportunidad... (Ruiz, Santiago y Castillo, 2018, p. 108). En contraste, al responder a la primera pregunta del cuestionario, los estudiantes activaron un conjunto amplio de estereotipos que se exponen a continuación: colegio, profesor, trabajo, actividad, obligación, casa, cuaderno, esfero, lápices, borrador, colores, libro, internet, diccionario, responsabilidad, deberes, esfuerzo, leer, consultar, investigar, estudiar, escribir, dibujar, aprender, pereza, tiempo, nota... (Ruiz, Santiago y Castillo, p. 108). 
Se presenta, además de lo expuesto, otra posibilidad en relación con el término problema, que toma forma en la relación tarea PLT problema. Probablemente, el estudiante encuentra en la tarea un impase, entonces una dificultad que genera algo adverso, que se opone a sus deseos o al uso de su tiempo libre. En este caso también se da cuenta de una conformidad con el protocolo semántico de tarea.

Lo anterior se completa con la presencia de una relación transgresiva que articula el término mejoramiento a la primera parte de la argumentación: la tarea genera problemas, contiene problemas pero lleva a / permite / contribuye a mejoramientos. Entonces, a pesar de lo adversa que puede resultar, de las dificultades que provoca, el estudiante considera que la tarea supone un mejoramiento.

\section{Tarea entonces obligación entonces recompensa}

Dos terceras partes de la población están a favor de esta cadena, con lo cual se valida la existencia de estas representaciones como conformes al protocolo semántico de tarea. Lo anterior significa que la ausencia, en el núcleo lexicográfico, del término recompensa no se presenta como una inconformidad sino como la introducción de una nueva asociación. Como ya se advirtió, esta entidad léxica traduce el término resultado -del núcleo lexicográfico- y se precisa en la palabra nota, en el núcleo reconstruido de las respuestas de los estudiantes.

Lo expuesto revela la idea de que la tarea se presenta como una obligación que produce una recompensa. Entonces, el grupo de estudiantes asume estos términos mediante relaciones normativas (por lo tanto) que establecen asociaciones de causa-efecto. La tarea se impone, por alguien, con miras a obtener una recompensa, que se traduce habitualmente en una buena nota, superar una mala nota o aprobar el año.

Los hábitos docentes, los discursos en la escuela y las estrategias a las que generalmente se recurre en el mundo educativo para lograr que las tareas se hagan han permeado el discurso, las creencias y las prácticas de los estudiantes. Esto contribuye, a mediano y a largo plazo, a formar ciudadanos con una visión utilitarista de la educación y a una búsqueda de recompensas por cada acción realizada o función asumida. Por estas razones, los estudiantes acogen este encadenamiento argumentativo como posible.

Tarea entonces dificultad entonces ayuda de los padres

Fundada sobre dos relaciones normativas, esta cadena introduce un nuevo elemento en el significado de tarea: ayuda de los padres. Se trata de una asociación no conforme al protocolo semántico de tarea. Ni en el núcleo lexicográfico ni en los estereotipos identificados en los diccionarios se encontró término alguno que remitiera a las formas padres o a (su) ayuda. En consecuencia, al estar de acuerdo con este encadenamiento, los estudiantes legitiman esta relación normativa que se establece entre la tarea que supone una dificultad, cuya superación requiere la ayuda de un tercero: los padres, especialmente.

El primer término de esta cadena argumentativa, dificultad, está adecuado al protocolo semántico de tarea, pues aparece en el núcleo lexicográfico. Con el acuerdo de un $66,57 \%$ de estudiantes, se ratifica la concepción de dificultad, como inherente a la tarea en el medio escolar. Sin embargo, esta entidad léxica no se moviliza en los bloques de significado del núcleo reconstruido a partir de las respuestas en los cuestionarios ni en los estereotipos de esta población de estudio.

La inclusión de los padres y de su ayuda en la concepción de la tarea, y de la aceptación de esta idea por parte de los estudiantes, devela las prácticas frecuentes en el sistema educativo en el que se llevó a cabo la indagación. Según esto, los profesores asignan a los estudiantes una serie de actividades, de trabajos, de ejercicios en cuyo desarrollo se espera o se obliga la participación de otros sujetos para que los asistan. Así, no resulta extraordinario escuchar frecuentemente de padres y de estudiantes quejas sobre el exceso en la asignación de tareas, en numerosas ocasiones desproporcionadas y sin sentido. 
Tarea entonces desmotivación sin embargo trabajo personal

El 53,13\%, aunque levemente superior al porcentaje de estudiantes que se mostró en desacuerdo con esta propuesta de encadenamiento argumentativo $(46,87 \%)$, pone de manifiesto una ausencia de decisión significativa, sobre la validez, o no, del encadenamiento propuesto. Esta similitud en las cantidades de respuestas posible-imposible se comprende si se precisa que se introdujo una entidad léxica que no se encuentra ajustada al protocolo semántico de la expresión tarea. El término desmotivación no tiene soporte alguno en el núcleo lexicográfico ni en el mismo núcleo reconstruido sobre la base de las definiciones de los estudiantes. Tan solo unos pocos estudiantes, en los estereotipos, relacionaron la tarea con entidades lingüísticas próximas a la desmotivación (esto se evidencia a través, por ejemplo, del uso de la forma coloquial mamera que tiene en este contexto el significado de aburrimiento, cansancio, fastidio).

Se podría esperar, pese a esta nueva asociación, un alto porcentaje de desacuerdos o de acuerdos, esto es, un mayor número de posible o de imposible, pero de ninguna manera esta suerte de equilibrio. Por lo tanto, esta cercanía en las consideraciones de los estudiantes pone de manifiesto justamente una ambigüedad en la tarea, en la medida en que esta remite, según las respuestas, a un trabajo, mayoritariamente personal, es decir, del estudiante. Entonces se puede establecer que es la relación transgresiva desmotivación SE trabajo personal la que determina este equilibrio, que sí induce en las prácticas a la desmotivación por parte de los estudiantes, producto de la obligación de desarrollar la actividad impuesta.

Lo anterior lleva a sugerir que la relación normativa tarea PLT trabajo personal estaría en completa conformidad con el significado lexicográfico y sería una manera de actualizar la representación reconstruida a partir de las definiciones de los informantes. Pero lo que se refleja en las respuestas similares de los estudiantes (posible 53,13\% e imposible 46,87 \%) es la transgresión tarea PLT desmotivación.
De este modo, la tarea genera un malestar, una reacción negativa que contiene una axiologización en la zona modal hedónico-afectiva. En consecuencia, esta secuencia argumentativa, aunque dividida entre lo posible y lo imposible, subraya lo que resiente un número importante de estudiantes frente a las actividades asignadas en las instituciones educativas.

Tarea entonces esfuerzo sin embargo aprendizaje

A diferencia de las reacciones similares encontradas en la cadena argumentativa anterior, el 93,27\% de los estudiantes consideraron como posible la relación propuesta, según la cual la tarea supone o exige un esfuerzo, pero se logra un aprendizaje. La aceptación de este encadenamiento revela unas asociaciones no conformes con el protocolo semántico de tarea. Esta no conformidad se establece más por la existencia de la relación transgresiva $\mathbf{S E}$ (sin embargo) que por los términos empleados. La entidad aprendizaje se ajusta al protocolo semántico en cuanto haría parte del resultado obtenido en la tarea. También lo es la palabra esfuerzo que se consolida, con los valores que activa, en la aceptación de esta cadena argumentativa.

Si bien la realización de una tarea demanda un esfuerzo a quien la ejecuta, el aprendizaje alcanzado gracias a las acciones implementadas y a las dificultades superadas es también un efecto o, en función del compromiso y de la voluntad del sujeto, la consecución de un objetivo. En consecuencia, el encadenamiento que da cuenta de esta asociación, según el significado lexicográfico, es tarea entonces esfuerzo entonces aprendizaje, en el que se marca claramente la relación normativa que actualiza una relación causa-efecto-fin. Lo anterior se explica por el hecho de que la tarea consiste en una actividad, en un trabajo y, como tales, actividad y trabajo exigen un esfuerzo para su realización y, en general, esta ejecución tiene un resultado que corresponde, en el ámbito escolar, a un aprendizaje.

Se puede observar en la secuencia tarea PLT esfuerzo PLT aprendizaje, acorde con el protocolo semántico, una orientación diferente a la que eligen los estudiantes, es decir, tarea PLT esfuerzo SE 
aprendizaje en la que se obtiene un resultado que no necesariamente es esperado, en el que no prevalece la voluntad del sujeto que realiza el trabajo asignado.

En conclusión, el que la mayoría de estudiantes acoja la posibilidad de esta argumentación, que no está acorde con el protocolo semántico de tarea, permite establecer que, en las prácticas escolares, en las actividades académicas en las que debe participar el estudiante, al menos en el contexto estudiado, se asume la tarea como un esfuerzo pero que no necesariamente conlleva un aprendizaje. Esto corresponde a una acción realizada por obligación en la cual se requiere el "empleo enérgico de la fuerza física contra algún impulso o resistencia" y el "empleo enérgico del vigor o actividad del ánimo para conseguir algo venciendo dificultades" (DRAE). Pese a ese ánimo por asegurar y a ese brío para vencer las dificultades, la mirada no se orienta hacia el aprendizaje. Este último se obtendría sin proponérselo, pues la voluntad está ocupada en franquear aquello que se ha convertido en obstáculo: la actividad asignada que da forma a la tarea.

Tarea entonces indisciplina entonces exigencia entonces nota

Con similares características a lo observado en las reacciones frente al quinto encadenamiento argumentativo, las relaciones propuestas en esta cadena reflejan porcentajes muy cercanos y ello a pesar de un ligero predominio de quienes aceptan la posibilidad de lo allí expresado. Esta débil diferencia en los porcentajes revela una indecisión en la apreciación que del encadenamiento tienen los informantes. Si se observa el núcleo lexicográfico, se puede encontrar que esta vacilación entre posibleimposible podría originarse en la inclusión de nuevos elementos en las asociaciones de tarea que no se encuentran en consonancia con el protocolo semántico de esta unidad léxica.

Los términos indisciplina y nota no se presentan en ninguno de los bloques de significado del núcleo. Tampoco existe palabra alguna de este significado léxico que motive la aparición de estas representa- ciones en el encadenamiento ni en las lecturas de los informantes. Por lo tanto, indisciplina y nota no se encuentran en el significado de tarea presentado por los diccionarios de lengua castellana, sino en las representaciones de los sujetos.

Se puede entender esta aceptación del carácter posible del encadenamiento $y$, también, la frágil diferencia posible-imposible, si se asume que la indisciplina se relaciona con la tarea porque aquélla hace difícil el desarrollo de las actividades académicas y pone en entredicho la autoridad o el control de los docentes. Como reacción a la no "observancia de leyes y ordenamientos" (DRAE), en principio, necesaria para los procesos de aprendizaje/enseñanza en clase, los profesores asignan tareas $y$, de esta manera, aseguran la exigencia para restablecer el orden. Para culminar, esta asociación - motivada por el contexto y la cultura escolar - fundada sobre conectores normativos se cierra con una consecuencia, de fuerte presencia en las prácticas educativas: el término nota y lo que ésta implica.

Como se señaló, la nota funge como un elemento fundamental de la tarea a tal punto que su activación en las definiciones de esta es numerosa y lleva a la inclusión de este término en el último bloque de la reconstrucción del significado léxico. La inserción de este término da cuenta del influjo del discurso escolar, especialmente el de los docentes, sobre las prácticas, los discursos y las representaciones que los estudiantes tienen de la tarea. Esto valida, en cierto modo, lo que se propuso en el estudio del núcleo reconstruido en el sentido de que cada vez más se refuerza la creencia y los hábitos de los estudiantes en el sistema educativo colombiano en torno a la relación aprendizaje-formación-compromiso y resultado-nota. Desde las prácticas de los actores educativos hasta sus discursos cotidianos, en colegios y escuelas, así como en hogares, se prepara a los ciudadanos, a los sujetos sociales, para unas relaciones y una vida sociocultural determinada y animada por el resultado, especialmente por el premio: la nota. 


\section{De las concepciones movilizadas en el discurso}

Con el ánimo de obtener una concepción más amplia de tarea y, al mismo tiempo, de corroborar los datos estudiados mediante las preguntas 1 y 3 del cuestionario, se solicitó a la población responder ante esta situación:

Lee cuidadosamente la siguiente situación y responde: En una institución educativa la Dirección tomó la decisión de quitar las tareas debido a las constantes quejas de padres y estudiantes. Marca con una $\mathrm{x}$ si estás de acuerdo o no con esta decisión: Sí:__/ No: __. ¿Por qué?

En principio, de un total de 712 participantes, 533 estudiantes $(74,85 \%)$ manifestaron su desacuerdo ante la supresión de las tareas escolares, mientras que 179 (25,14\%) se mostraron de acuerdo con su abolición. En los apartados que siguen se ofrece el análisis de los argumentos que los estudiantes presentaron para justificar su rechazo a la eliminación de las tareas. Para ello, se exponen a continuación los aspectos cuantitativos más sobresalientes que emergen de las respuestas de la población. Las entidades léxicas que aparecen en la tabla 2 corresponden a los aspectos nucleares de los argumentos ofrecidos por los estudiantes, por lo tanto, presentan los términos (18) más representativos que esgrimieron como elemento fundamental para oponerse a la eliminación de la tarea.

Tabla 2. Argumentos a favor de las tareas escolares

\begin{tabular}{lcc}
\hline $\begin{array}{l}\text { Términos base de las } \\
\text { argumentaciones }\end{array}$ & $\mathbf{5 3 3}$ & \% \\
\hline Aprender / aprendizaje & 260 & 48,78 \\
\hline Sacar notas & 70 & 13,13 \\
\hline $\begin{array}{l}\text { Entender / } \\
\text { entendimiento / } \\
\text { comprender }\end{array}$ & 51 & 9,57 \\
\hline $\begin{array}{l}\text { Reforzar / refuerzo } \\
/ \text { reforzamiento }\end{array}$ & 42 & 7,88 \\
\hline $\begin{array}{l}\text { fortalecer / } \\
\text { afianzamiento }\end{array}$ & 41 & 7,69 \\
\hline Hacer & 37 & 6,94 \\
\hline Tiempo & 34 & 6,38 \\
\hline Ayuda(r) & & \\
\hline
\end{tabular}

\begin{tabular}{lcc}
\hline $\begin{array}{c}\text { Términos base de las } \\
\text { argumentaciones }\end{array}$ & $\mathbf{5 3 3}$ & \% \\
\hline Investigar & 30 & 5,63 \\
\hline Profundizar & 27 & 5,07 \\
\hline Enseñar / enseñanza & 27 & 5,07 \\
\hline Conocimientos/conocer & 25 & 4,69 \\
\hline Estudio & 24 & 4,5 \\
\hline Repasar & 23 & 4,32 \\
\hline Practicar / aplicar & 20 & 3,75 \\
\hline Mejorar & 15 & 2,81 \\
\hline Desarrollar & 13 & 2,44 \\
\hline Esforzar (se) / esfuerzo & 10 & 1,88 \\
\hline Saber & 10 & 1,88
\end{tabular}

En los siguientes apartados se presenta el análisis de los aspectos que los estudiantes señalan para justificar la no supresión de las tareas, los cuales se estructuran en torno a los términos: aprender / aprendizaje, sacar notas, reforzar, hacer, tiempo, ayudar e investigar.

\section{Tarea escolar PLT aprender / aprendizaje}

533 estudiantes manifestaron su desacuerdo con la supresión de las tareas. Las razones que este grupo expuso presentan gran variedad, no obstante, (ver tabla 2) el argumento que predomina involucra los términos aprender/aprendizaje. Al respecto, 260 estudiantes coincidieron en señalar que no se deben suprimir las tareas puesto que estas permiten aprender o facilitan el aprendizaje.

De este grupo, 105 sujetos sostienen que no están de acuerdo con la supresión de las tareas porque estas se constituyen en un medio para aprender, específicamente, sobre las temáticas que se han trabajado en el aula de clase. En relación con esto, 64 informantes señalan que al eliminar las tareas no tendrían la posibilidad de aprender; para este subgrupo, entonces, suprimir las tareas equivale a no aprender, lo cual ratifica lo planteado, en el sentido de que la tarea viabiliza el aprender y su eliminación conlleva un efecto negativo. En la misma dirección, 35 estudiantes sustentan su desacuerdo dado que la tarea permite aprender más. 
Con base en lo señalado, se tiene el encadenamiento tarea PLT aprender / aprendizaje, que coincide con el segundo bloque de significado del núcleo de la entidad léxica, según los estudiantes. Cabe señalar que en ese bloque se hace presente el carácter epistémico de la tarea, en la medida en que se erige como recurso que brinda la posibilidad de obtener conocimientos, así como ampliar los ya adquiridos, lo cual implica que se le asocien valores pragmáticos, intelectuales y hedónico-afectivos positivos. Esto permite inferir que, para esta población, la tarea es una actividad útil en la medida en que implica aprender, lo cual la hace interesante y que genera satisfacción pues contribuye al crecimiento cognitivo, personal y sociocultural.

A primera vista, se trata de una sólida justificación pues los niños y jóvenes ponen de manifiesto la importancia que tiene la tarea como un medio que lleva a aprender. De esto se pueden identificar valores positivos en la zona modal axiológica, así como valores epistémicos. Sin embargo, se hace necesario aproximarse a algunos de los discursos de los estudiantes para precisar esta tendencia en favor de la continuidad de las tareas debido a su aporte al aprendizaje. En un primer momento, se advierte esta orientación tareas PLT aprender / aprendizaje en casos como:

- "no, porque gracias a las tareas realizamos aprendizaje cada día"

- "no, porque no sea muy cómodo para nosotros los estudiantes las tareas son necesarias ya que nos ayudan a aprender y reforzar los temas vistos en clase"

- "No, aunque las tareas son fastidiosas y quitan el tiempo libre de los estudiantes son muy importantes para aprender"

- "no, porque así tenemos que hacer en casa y podemos aprender más por nuestra propia cuenta"

- "no, porque una tarea, aunque sea aburrida o nos dé pereza son necesarias ya que de ellas aprendemos o reforzamos un tema o varios"

En varios ejemplos de los discursos expuestos se observa un predominio de términos que activan una axiologización positiva que realza el efecto de la tarea, esto es, el aprendizaje / aprender. Ayudar a, importante y bien son entidades léxicas que movilizan valores pragmáticos positivos que refuerzan los valores intelectuales positivos contenidos en aprender / aprendizaje.

Se identifican también algunos aspectos ligados a la zona modal ontológica puesto que, al defender la tarea como una necesidad, se activan valores aléticos. En este orden de ideas, importantes y necesarias son términos que contienen esta orientación ontológica. Entonces, no se trata solamente de la tarea como una práctica buena que ayuda a lograr algo, sino también como una actividad necesaria. A esto se agrega la utilidad que justifica la continuidad de la tarea; lo anterior se hace evidente en la expresión servir para, a lo que se agregan estas variantes de lo positivo expresadas así: "se puede atrasar y eso sería muy malo". Estos términos contienen valores pragmáticos positivos e inclusive ético-morales positivos. Esto lleva a concluir que la tarea es buena, necesaria y útil para los estudiantes encuestados.

Lo anterior debe ponerse en contraste con los datos encontrados en otros apartados del cuestionario (preguntas 1 y 3 ), pues se debe tomar en consideración la representación que los estudiantes tienen de aprender/aprendizaje, de aquello que se aprende, de las razones y fines de este aprendizaje, así como de la relación tarea PLT aprendizaje PLT nota. Esto significa que el aprendizaje no se presenta como un fin per se sino como un medio que lleva a la obtención de una recompensa: la nota o calificación. Ya se expresó en otros apartados la posible razón de la emergencia de esta idea utilitarista de tarea y aprendizaje con miras a una nota (positiva), así como las implicaciones para la vida individual y sociocultural de los estudiantes y futuros ciudadanos.

Lo anterior muestra que, como consecuencia de las experiencias, prácticas y acciones escolares, así como de los discursos que circulan entre escuela y hogar, el estudiante ha incorporado a sus concepciones, relacionadas con la educación, la evaluación y específicamente con la tarea, una visión distorsionada del aprendizaje. De estas concepciones se des- 
prende un activismo sin fundamento que se limita a ejecutar acciones, a desarrollar ejercicios, a copiar información, a cumplir con una actividad asignada aparentemente para favorecer el aprendizaje. Pero, realmente, a partir de los diversos datos recogidos, se asiste a un aprendizaje sin propósito, en el que el papel del estudiante se desdibuja en el recorrido que lo lleva a alcanzar el éxito académico, que se reduce a obtener una nota que permita aprobar un período escolar, sin que exista un aprendizaje conducente al mejoramiento de las dimensiones que lo constituyen como sujeto, lo que se evidencia en una muy baja frecuencia en la activación de términos como mejorar / mejoramiento, desarrollar, saber, entre otros (cfr. Tabla 2).

\section{Tarea PLT sacar notas}

Para otro grupo representativo (70), la razón para la no erradicación está sustentada en que la tarea es útil porque se instaura como fuente de notas que se tornan en importantes para obtener la aprobación de las asignaturas, del periodo y del año escolar. En este caso deja de tener relevancia el aprender y se vuelve central el resultado cuantitativo que se deriva de su realización.

De acuerdo con lo anterior, un subgrupo de estudiantes (22) argumenta que no se deben eliminar las tareas porque la razón de ser de éstas es sacar notas. Según esto, la tarea se convierte en un medio con el que cuenta el docente para evaluar cuantitativamente al estudiante. Ahora bien, sacar notas tiene como consecuencia, entre otras: pasar el año, pasar la materia, ganar el periodo, mejorar notas, subir puntos, mejorar el promedio, calificar al estudiante. Esto implica que, si se suprimen las tareas, se reducirían las notas, por lo tanto, se afectaría negativamente, no el aprendizaje del estudiante, sino su tránsito por las distintas instancias del sistema escolar. Se deduce de ello una concepción de evaluación centrada en lo cuantitativo y punitiva, en la que prima el producto mas no el proceso, en la que no interesa la formación del sujeto sino su medición y, a partir de ésta, su promoción.
Se tiene, en este caso, el encadenamiento tarea PLT notas que está en consonancia con el tercer bloque del núcleo del significado de tarea. Se desdibuja aquí el carácter epistémico e intelectual de ésta, dado que ya no se ve como medio para obtener conocimiento sino en recurso para obtener una certificación escolar, quedando relegada a un segundo plano la formación del sujeto. Se manifiestan, por lo tanto, valores de orden pragmático y hedónicoafectivo positivos: la tarea es útil en la medida en que suministra notas para que el estudiante obtenga la aprobación y ello, desde luego, genera sentimientos positivos.

Como se enunció en el apartado anterior, aunque de manera aparente el encadenamiento argumentativo tarea PLT aprendizaje / aprender da cuenta de la razón principal que se opone a la supresión de la tarea, existen otras motivaciones, unas justificaciones menores en cantidad, pero sumamente importantes, que ponen de manifiesto la orientación instrumental que se le asigna a esta actividad escolar.

Se puede precisar que la nota no es en sí misma una problemática de los procesos de aprendizaje / enseñanza ni de la educación. Es realmente el valor que se le asigna, el estatus que se le ha conferido y las prácticas que se han instaurado entre los actores como producto de su influencia. Es en este sentido que el grupo de estudiantes ofrece sus razones para oponerse a la hipotética supresión de la tarea.

La relación normativa tarea escolar PLT (sacar) nota cobra, de esta forma, una alta importancia entre los argumentos de los estudiantes, como se observa en las siguientes respuestas:

- "no, porque de esas notas pasas"

- "no, porque es bueno y nos da notas"

- "a uno le ayuda para sacar buenas notas"

- "también nos ganan las notas para ganar el periodo"

- "tenemos más puntos en la nota final"

- "para sacar una buena nota"

- "la tarea cuenta como una nota para pasar la materia correspondida" 
- "no, porque nos perjudica en el sentido de notas"

- "sin tareas tendríamos menos calificaciones"

- "es obtener una nota beneficiaria que compute las notas o mejoren un promedio"

En los términos "pasas", "bueno", "nos da", "ayuda para", "buenas", "ganan/ganar", "más (puntos)”, "nos perjudica", "tendríamos menos", "beneficiaria”, "mejoren", se advierte el carácter de lo útil, llevado a lo meramente instrumental. Se trata, por lo tanto, de la primacía de lo utilitarista, esto es, de una orientación pragmática sobre los valores intelectuales y epistémicos. Se valida, en estas respuestas, la secuencia argumentativa tarea escolar PLT aprendizaje PLT nota, que contiene los términos de mayor frecuencia en la argumentación de los estudiantes frente a la supresión de las tareas. Lo que prima es, en consecuencia, la voluntad o la intención de conseguir una cuantificación de los resultados, traducidos en nota, con miras a "ganar el año". En este orden de ideas, sumados a los valores axiológicos positivos (pragmáticos) se activan otros de la zona modal finalizante: los volitivos.

Al observar los núcleos, el lexicográfico y el que resulta de las definiciones de los estudiantes, se evidencia que el primero incluye, en el último bloque de significado (resultado) de tarea escolar, los términos de mayor ocurrencia en los discursos de los estudiantes: aprendizaje y nota. El segundo contiene las dos entidades léxicas objeto de estudio aquí: aprendizaje PLT nota. Esta observación revela claramente que los argumentos de los estudiantes son coherentes con el protocolo semántico de tarea, pero glosan la calidad y el tipo exacto del resultado. Además, en el núcleo reconstruido, estos términos se establecen en un vínculo normativo que realza la relación medio-fin. En este orden de ideas, el aprendizaje es un medio para la obtención de un propósito: la nota. De esta manera, se valida la orientación pragmática asociada a valores volitivos, en la que la nota se torna en la meta por alcanzar; para ello se recurre a la tarea que, aparentemente, genera aprendizaje.
Como se ha señalado, el discurso de los estudiantes en la defensa de la tarea dista significativamente de aquello que ellos mismos pueden generar. Son las prácticas socioculturales, reforzadas y legitimadas por la escuela, las que han contribuido a la aparición de estas concepciones, así como a sus discursos y a sus prácticas. Esto lleva a establecer como hipótesis que la experiencia educativa ha incidido en las representaciones de los estudiantes.

\section{Tarea PLT entender / entendimiento / comprender}

En los discursos de los estudiantes se encuentran otros argumentos relacionados con entender/comprender, que parecen ser más ajustados a algunas de las prácticas efectivas en el salón de clase y fuera de la institución educativa. En principio, la tarea que el docente asigna le permite al estudiante alcanzar una comprensión o un entendimiento de diversos aspectos tratados en clase. Quizá se trata de operaciones cognitivas que efectivamente toman lugar en el proceso formativo del estudiante. En este sentido, la tarea lograría alcanzar una función valiosa para el estudiante: asimilar, explicar, así como alcanzar conocimiento de situaciones, procesos, temáticas, percibir relaciones, identificar causas y efectos, entre otros aspectos. Algunos elementos, según las respuestas de los estudiantes, permiten precisar y validar lo expuesto:

- "no, porque las tareas nos ayudan a entender más las materias y a manejar el tema visto en clase"

- "no, porque no entenderíamos nada"

- "no, entonces como entenderíamos las cosas que nos explican los profesores"

- "no, porque la tarea es otra forma de entender lo aprendido o dicho por los demás"

- "no, porque las tareas nos ayudan a entender mejor las cosas a que nos queden grabadas en la cabeza"

Estos segmentos revelan una concepción concreta de una de las funciones de la tarea escolar: entender/comprender tema / cosa / materia. El objeto de esta comprensión corresponde especialmente a temas, informaciones, contenidos, datos. 
Es claro que esta orientación de la tarea es necesaria, pero es insuficiente, pues en las respuestas de los estudiantes no se activan entidades léxicas que contengan representaciones sobre operaciones mentales, aplicaciones, verificaciones.

Lo expuesto deja entrever que, aunque -en apariencia- predominen valores de la zona modal de juicios de verdad (epistémicos) y que éstos estén estrechamente relacionados con los axiológicos positivos (intelectuales), inclusive con los pragmáticos, no son suficientemente sólidos y su alcance es limitado. Dicho de otra manera, el conocimiento, visto como tema, materia, cosas, contiene valores epistémicos que resultan, probablemente, interesantes para los estudiantes, de ahí que el aprehenderlos les sea útil. Pero, como se estableció atrás, esto no parece dar cuenta de otros componentes que serían objeto del entendimiento. Además, se podría pensar que priman, ante todo, contenidos de orden declarativo, lo que lleva a que el aprendizaje se asuma como simple memorización, con lo cual queda en entredicho la comprensión y el entendimiento, dejando de lado - o relegados a un segundo plano- contenidos de orden procedimental y condicional.

Otro aspecto que sobresale en estos ejemplos es que no solamente el estudiante se beneficia de la presencia de la tarea, sino también el docente, pues el trabajo que éste impone contribuye a la comprensión de los contenidos tratados. Parece importante esta concepción, pero no bastaría con esa mirada sobre la tarea y, por lo tanto, se requeriría una mejor comprensión, una conceptualización, un diseño, una ejecución, un seguimiento y una evaluación-mejoramiento de aquélla. A manera de ejemplo, el docente podría concebir tareas con miras a verificar los aprendizajes, a identificar estrategias, a cualificar operaciones, entre otros objetivos. Ahora bien, cada actividad asignada debería resultar de metas definidas y tener propósitos concretos, estar sustentada por razones sólidas, así como observar una metodología clara y consistente.

En pocas palabras, esta argumentación de la importancia de la tarea está a medio camino en la concepción tanto de los docentes como de los estudiantes. Y son finalmente estos últimos quienes conciben estas características a partir de sus vivencias escolares, de las costumbres de los maestros y, por supuesto, de sus discursos, anclados en un sistema educativo que ha otorgado un valor excesivo, y distorsionado, a un sistema de calificación - notas-, en el que prevalece la cifra sobre los criterios de evaluación y los propósitos formativos de la educación misma.

Tarea escolar PLT Reforzar / refuerzo / reforzamiento / fortalecer / afianzamiento

La categoría emergente de estos argumentos pro-tarea escolar, identificada bajo la etiqueta reforzar, está constituida por entidades léxicas como: refuerzo, reforzamiento, fortalecer y afianzamiento. Para determinar con claridad esta serie de argumentaciones de los estudiantes, se presenta a continuación algunas de las secuencias discursivas que se identificaron en sus respuestas:

- "no, porque la tarea es como un refuerzo de lo de clase"

- "la tarea es un refuerzo para nuestros aprendizajes"

- "la tarea escolar es importante porque se refuerza lo aprendido"

- "no, porque las tareas son un mecanismo de refuerzo [...] sobre lo que se aprende en la clase"

- "no, ya que las tareas pueden ayudar a reforzar los temas que no se entiendan en clase"

- "reforzar lo que se aprende en el colegio"

- "no, porque la tarea refuerza lo visto en clase"

Existe, de acuerdo con esto, un predominio de la atención centrada en contenidos y de esta manera se confortan valores epistémicos e intelectuales positivos. Sin embargo, estos contenidos, en la mayoría de los casos, se limitan a una serie de datos desarticulados que no alcanzan integralidad ni unidad y que, por lo mismo, corresponden a un saber declarativo, desprovisto de análisis y reflexión.

Con estas secuencias discursivas se refuerza la representación de la tarea como una actividad que facilita la consolidación de algo con miras a 
lograr algo: consolidar el entendimiento de temas para un aprendizaje que lleve a conseguir buenos resultados. Lo anterior activa valores pragmáticos positivos, pero apuntan a utilidades puntuales, de corto alcance y a corto plazo, y que hacen circular una idea de educación para el éxito, una educación a medias, una educación insuficiente o inconclusa, esto en el plano de sus ambiciones sociales como institución fundamental. Es claro que pretender que la tarea es importante por su aporte al refuerzo de temas e informaciones no es necesariamente negativo, pero sí su carácter insuficiente, incompleto e instrumental.

\section{Tarea escolar PLT hacer}

En este caso, los argumentos se enfocan, en principio, en una etiqueta verbal que genera ideas relacionadas con la acción, con la activación del dinamismo, de la capacidad propositiva y transformadora tanto del sujeto que aprende como de aquél que enseña. Ahora bien, se debe identificar de qué tipo de hacer, de qué acción se trata exactamente, de manera tal que se logre caracterizar claramente qué discursos de los estudiantes prevalecen, por una parte, y están permeados por los de los docentes, por otra, a lo que se debe agregar cómo develan las concepciones que se construyen gracias a las prácticas escolares que se naturalizan como válidas y como únicas.

La lectura juiciosa de los enunciados expuestos a continuación permite encontrar algunos rasgos propios de los discursos que circulan en las instituciones escolares e incluso en los contextos familiares:

- "no, porque los niños tenemos que investigar y si no tenemos nada que hacer en la casa que haga la tarea"

- "no, porque no tendrían nada que hacer los estudiantes en las tardes o en la mañana"

- "no, porque sería quitarle algo que hacer a los niños en las tardes"

- "nos queda demasiado tiempo en la casa $\sin$ hacer nada"

- "veces hay tiempos libres y no hay nada que hacer"
- "no, porque sin tareas los estudiantes no tendrían más compromiso hacia el estudio tendrían más tiempo libre sin nada que hacer"

- "algunos estudiantes no tienen que hacer nada en sus casas"

Las razones de los estudiantes revelan básicamente un hacer en relación con una dimensión espaciotemporal y la ausencia de actividad. Esto significa que no están presentes las precisiones relativas a aquello que se hace en el marco de la tarea.

Según los datos recogidos, se concluye que la tarea es una necesidad que concierne al menos a dos de los actores educativos. Padres de familia y estudiantes se benefician de la continuidad y de la asignación de las tareas, por parte de la escuela, en la medida en que, para los primeros, se garantiza que sus hijos no van a hacer un mal uso de su tiempo libre o simplemente no van a tener tiempo libre. Ante la ausencia de tareas no habrá nada qué hacer, lo que provocará consecuencias negativas para los estudiantes y, por supuesto, para los acudientes. Por lo tanto, se consolidan, gracias a estas argumentaciones, valores de la zona modal axiológica (pragmáticos): la tarea es útil porque contribuye a ocupar a los estudiantes de tal manera que hagan algo, especialmente en la casa. También se activan valores de la zona modal ontológica, en los que va a primar la necesidad de la tarea para resolver situaciones sin la cual los padres difícilmente podrían asegurar, o imaginar asegurar, el control de sus hijos y de su tiempo libre.

Esa necesidad y esa utilidad están ligadas a la dimensión temporal representada por "tardes", "mañana", "demasiado tiempo" y "tiempo libre", entre otras entidades léxicas. Igualmente se asocian con la dimensión espacial que se hace presente mediante la forma léxica "casa". En consecuencia, se refuerzan las dos zonas modales en los valores específicos contenidos en, y relacionados con, las concepciones de tiempo y espacio.

Ocupar el tiempo libre en casa y evitar el tener nada que hacer concretan esta asociación tarea escolar PLT hacer. En principio, se puede creer que es una orientación, una idea de justificación de la tarea 
que da cuenta de representaciones que favorecen la formación de los estudiantes y del papel de padres y docentes. Sin embargo, la carencia de etiquetas nominales, por ejemplo, que complementen el verbo hacer, que delimiten su significado, sugiere la existencia de un terreno amplio y vago en la noción de tarea y en las prácticas que le subyacen. Esto lleva a considerar que, como se adelantó en otro apartado, a la tarea la caracteriza la acción per se, el activismo desprovisto - tal vez- de razones sólidas y de propósitos definidos.

Si se retoman elementos del núcleo del significado lexicográfico de tarea, sólo un término de éste se retoma en las argumentaciones de los estudiantes: tiempo. Pero existe una diferencia significativa dado que, en el núcleo lexicográfico, tiempo está acompañado de un modificador: limitado, mientras que en las justificaciones de los estudiantes la modificación es completamente opuesta: libre y demasiado.

Lo anterior se puede expresar como sigue: tarea PLT tiempo limitado (núcleo lexicográfico) y tarea PLT tiempo libre y demasiado tiempo (secuencias discursivas en las argumentaciones de los estudiantes). Se advierte un cambio en el eje axiológico de tiempo: pasa del polo negativo (pragmático) a una orientación ambivalente. Es tal vez esta modificación la que preocupa, incluso a los mismos estudiantes, porque lo que en principio es positivo y útil, tiempo libre, puede transformarse en una dimensión en la que se desarrollan acciones negativas, inapropiadas o inconvenientes. Entretanto, el estudiante introduce la idea de exceso en la dimensión temporal, lo que a su vez activa valores negativos, especialmente cuando no habría nada previsto por hacer en ese período y que conlleva la ejecución de actividades ligadas a conductas inadecuadas: "estaríamos en la calle vagueando", "es una forma de que los niños y niñas no entren en vicios ya que con las tareas se les ocupa más el tiempo" y "los niños y niñas por la tarde van a callejear o no hacer nada en la casa".

En relación con la dimensión espacial, los estudiantes agregan, a los elementos del núcleo lexicográfico, la entidad léxica casa como único referente del lugar en el que se desarrolla la tarea. Mientras que en los diccionarios no hay mención de ningún referente espacial, tanto en la definición de los estudiantes como en sus justificaciones, la realización de la tarea se limita a la casa. Se modifica, entonces, la representación de los contextos en los que se puede ejecutar esta actividad escolar. Se deduce, de ello, que en general la tarea se puede llevar a cabo en diferentes espacios y no está vinculada necesariamente a la casa y a los integrantes del círculo familiar. Esta variación en la representación de tarea vinculada al término casa se origina probablemente en el influjo de los hábitos y discursos de docentes y de padres de familia. Según esto, la amplia gama de posibilidades que tiene lo espacial en los diccionarios se reduce, entre estudiantes, exclusivamente al hogar, como espacio no solamente social sino vital.

Por último, se observa otra mirada reduccionista sobre la concepción de tarea en el primer bloque de significado del núcleo lexicográfico: de "obra y trabajo obligatorios" se deriva en un hacer ambiguo e impreciso que puede ser complementado con toda suerte de acciones y de propósitos, que no necesariamente obedecen a soportes teóricos que contribuyan a una formación genuina de los estudiantes.

En los párrafos anteriores se abordaron y estudiaron los términos base de las argumentaciones de mayor frecuencia en las respuestas de los estudiantes. De ahí que el análisis se centró en los argumentos cuya base es aprendizaje, (sacar) nota, entender/comprender, reforzar/refuerzo y hacer. Se anunció, al comenzar este apartado, que el término de mayor ocurrencia no superaba el $50 \%$ de los 533 estudiantes que se opusieron a la supresión de las tareas. Esta característica cuantitativa arroja luces sobre la diversidad y la dispersión de argumentos, lo cual devela la ausencia de marcas mayoritarias en las representaciones identificadas gracias a este componente del cuestionario administrado.

Lo anterior precisa que solamente cinco términos se presentan como los más representativos a pesar de la baja frecuencia en las argumentaciones de los informantes. Entonces, dada la dispersión de la gran mayoría de argumentos, clasificados en función de los términos base de cada uno de ellos, en lo que 
sigue de este análisis se procede a estudiarlos en su conjunto. Este amplio conjunto de entidades léxicas evidencia la multiplicidad de argumentos que tienen los estudiantes para defender la causa de la tarea. Ahora bien, un número tan alto de argumentos diversos no logra una relativa unidad, a pesar de algunas concepciones ya analizadas, sino que deja ver que no existe una visión consolidada de la importancia de esta actividad escolar.

Tarea escolar PLT ayuda(r), investigar, profundizar, enseñar/enseñanza, conocimientos/conocer, estudio/estudiar, repasar/repaso, practicar/aplicar, mejorar, desarrollar, esforzarse/esfuerzo, saber

En este punto se hace imprescindible establecer una aclaración que permita 1) agrupar una serie de entidades léxicas base de un conjunto amplio de argumentos y 2) comprender sus implicaciones en este análisis.

Se observa en estos términos algunos elementos que se complementan y, además, refuerzan y explican otros ligados a las cinco primeras entidades léxicas estudiadas. En este grupo de argumentos se pueden generar unos subgrupos alrededor de los objetos de las acciones que los estudiantes ejecutan gracias a las tareas.

El primer conjunto emergente se construye en torno a contenidos, informaciones y datos, términos que se asocian a tema. Es así como los estudiantes estiman que la tarea no debe erradicarse porque funge como ayuda, o simplemente ayuda a lograr algo: "entender los temas", "memorizar" ("tema", "ejercicio"). En la misma orientación se encuentra el término profundizar que se declina en profundizar "(a fondo) tema", "los temas de clase". De este subgrupo hace parte también repasar que se complementa con "tema (visto)", "lo aprendido (en clase)". Esta serie de argumentos se complementa con la inclusión del término base practicar "tema", "lo entendido", "lo que aprendemos", "lo visto en clase".

Las ilustraciones expuestas se relacionan estrechamente con dos de los términos de mayor frecuencia ya analizados: comprender/entender y reforzar, especialmente por la precisión que les otorga otro término en el cotexto, que en este caso corresponde a tema y sus variaciones. Se resalta entonces la consolidación de valores epistémicos e intelectuales positivos, pero sin desestimar las precisiones sobre las insuficiencias que ya se señalaron.

La orientación que apunta a los contenidos de los cursos, de las materias o asignaturas que configuran los planes de estudio de las instituciones escolares, se refuerza con la movilización de argumentos construidos sobre la base de investigar, estudiar y conocer/conocimiento. De esta manera, se consolidan los valores epistémicos e intelectuales. El seguimiento de los segmentos discursivos fundados en las entidades léxicas citadas determina que su cotexto lo constituyen términos como investigar más, tema -por una parte-, conocer más, información, tema -por otra- y, finalmente, estudiar mucho menos, en casa, más (a fondo el tema), lo aprendido. En ciertos casos, investigar, conocer/conocimiento y estudiar, en su cotexto, no se complementan con término alguno, v. gr.: "no, porque las tareas sirven para investigar"; "no, porque también debemos estudiar", "nos ayuda a conocer", "adquiriendo más conocimientos" y "ampliar el conocimiento".

Se reitera en los dos tipos de complementos de investigar, estudiar y conocer/conocimiento (marcado lingüísticamente y con ausencia de marcador lingüístico), especialmente, la importancia de la tarea por su aporte a la consecución de contenidos, es decir, a la adquisición y a la ampliación de conocimientos, de los saberes declarativos de obligatorio tratamiento en la educación, particularmente en los tres ciclos consultados. Sin embargo, se hace necesario señalar que esta idea de adquisición, quizá de memorización de informaciones, ha permeado en las representaciones que los estudiantes tienen de las entidades léxicas, y de sus prácticas reales, como investigar y estudiar. En este orden de ideas, una actividad de consulta, de lectura, un ejercicio de repetición para la memorización, aparecen, según los datos obtenidos, como equivalentes de estudio y, lo que parece más complejo, de investigación.

Pese a los aspectos negativos expuestos, en el verbo estudiar se anuncian características que contribuyen de forma efectiva a asignarle a la tarea 
unas representaciones positivas y, evidentemente, existentes en las prácticas educativas. A esta entidad léxica se suman enseñar y desarrollar. Estas representaciones no se limitan a los conocimientos, a los saberes declarativos, sino que amplían las dimensiones en las que incide la tarea. Por lo tanto, se encuentran argumentos que insertan en las concepciones formas como enseña a ser responsable, orden y disciplina; desarrollar el cerebro, mejor capacidad mental, nuestras habilidades.

Estas entidades léxicas enriquecen las limitadas representaciones que se han expuesto a lo largo de este apartado. Con su aparición en el discurso de los estudiantes se aportan nuevos estereotipos al significado léxico y, por lo tanto, se refuerzan algunos valores y se activan otros. La zona modal axiológica se refuerza, específicamente en lo atinente a valores pragmáticos e intelectuales, así como la zona de juicios de verdad en lo que se refiere a valores epistémicos. Además, dada la importancia otorgada a la formación del sujeto, se activan valores ético-morales positivos. Ahora bien, la incidencia de estos refuerzos y de la activación no es significativa, si se tiene en cuenta que el número de argumentos que contienen estos términos base no alcanza un porcentaje importante, es más, aparecen entre los de menor frecuencia presentes en las secuencias discursivas.

Por último, si se suprimiera la tarea, el estudiante no lograría saber más o quizá culminaría los cursos sin saber nada. Como resultado de esta situación, vinculada a la entidad léxica saber, no se podría mejorar, en general, ni se mejoraría el rendimiento. Con estos dos términos se completa el amplio espectro de argumentos que, sin ser mayoritarios, ponen de relieve la ausencia de representaciones consolidadas y, aunque aportan elementos a los argumentos de mayor frecuencia, su incidencia es menor. Se advierte una preocupación por la eventual eliminación de la tarea pues haría mella en el saber y en el mejoramiento del sujeto, incluido el del conocimiento y el de los resultados, lo cual genera una consolidación de valores epistémicos y pragmáticos positivos.
Finalmente, la unidad lingüística esfuerzo/ esforzar(se), y su débil presencia entre los argumentos a favor de la continuidad de la tarea, sugiere vínculos significativos con los otros términos base de los argumentos expuestos para apoyar la tarea. Es difícil alcanzar resultados (memorizar contenidos, aprender temas, obtener buenas notas), por instrumentales que éstos sean, sin la implicación del sujeto que aprende, la cual supone esfuerzo. Algunos estudiantes lo expresan como "es mejor tener tareas para esforzarnos cada día más" y "necesitamos esforzarnos". Claro que con esta frágil frecuencia de argumentos construidos a partir del término esfuerzo/esforzar(se) se evidencia muy poca incidencia de valores hedónico-afectivos negativos. Esta evidencia consolida la ya clara modificación de elementos del núcleo, en la medida en que el elemento del núcleo lexicográfico, esfuerzo, se debilitó en las representaciones de los estudiantes.

\section{A manera de cierre}

Al revisar y analizar los resultados referidos a las respuestas de los estudiantes con respecto a las secuencias argumentativas propuestas, se puede ver que estos reforzaron varios de los nuevos estereotipos acordes o no con el significado léxico de tarea escolar, por un lado, y elementos del núcleo, por otro; esto teniendo en cuenta el núcleo y los estereotipos que se establecieron para tarea escolar de acuerdo con el discurso lexicográfico (véase Castillo, Santiago y Ruiz, 2018, pp.118-119). Asimismo, consolidaron las visiones de la tarea en la distorsión y en la ambigüedad. Prevalece, en las respuestas a los diferentes componentes del estudio, una visión marcada por la orientación pragmática, con predominio de un sentido utilitarista, y el interés en integrar a sus conocimientos una serie de saberes declarativos que favorezcan el alcance de una recompensa académica. Posiblemente, más tarde, esta manera de asumir las tareas, y la educación, tenga incidencias desfavorables en la vida individual y social. En el presente, se advierte una desorientación y una distorsión en la razón de ser de las tareas y en su posible función formativa en el desarrollo de niños y jóvenes. 
En relación cercana con esta primacía del resultado pretendido, transformado en una nota, y quizá más tarde en recompensas sociales, se advierte la variedad de estereotipos que se consolida con la multiplicidad de formas modales utilizadas para argumentar la oposición a la eliminación de las tareas. En este orden de ideas, en las justificaciones de los estudiantes para manifestar su acuerdo con la continuidad de estas prácticas escolares, recurrieron a términos base de la argumentación de la más diversa índole. Tal dispersión de argumentos es coherente con la diversidad de estereotipos identificados en las respuestas de los estudiantes. Se hace indispensable precisar que los números elevados de estereotipos y de argumentos no son en sí un aspecto negativo, sino la ausencia de mayorías significativas que permitan establecer, con mejor solidez, las tendencias aún más sobresalientes. Esta profusión de argumentos se dispersa, entonces, en múltiples direcciones, lo que conlleva a establecer que las miradas sobre la tarea escolar son muy disímiles y que, a pesar del influjo de las figuras docente y familiar, la idea de tarea no se ha consolidado en una imagen clara, mayoritaria y coherente.

$\mathrm{Al}$ abordar de manera específica los hallazgos ligados a los encadenamientos propuestos, se verifican nuevos elementos en el significado de tarea escolar como la fuerza proyectada por las representaciones de los estudiantes: discursos que son producto de la influencia de la acción discursiva de la vida escolar y de las experiencias que consiguen modificar los imaginarios, al igual que las acciones de los jóvenes colombianos. Más allá de la competencia semántica argumentativa que revelan los estudiantes en sus elecciones, posible-imposible, sus respuestas ponen de manifiesto percepciones y valoraciones que se originan en las prácticas escolares y que han distorsionado las concepciones de algunas actividades escolares, como la tarea. A su vez, esta constatación reitera algunas situaciones que ponen en tela de juicio la orientación de la educación, al menos en el contexto de este estudio, y el influjo que ha calado en la axiología de los futuros ciudadanos.
Por su parte, lo atinente a los discursos sobre la continuidad de las tareas demuestra un alto número de razones diversas, pero sin una mayoría definida, ni consolidación ni unidad, que los estudiantes esgrimen para defender la continuidad de la tarea como una práctica escolar necesaria y útil.

El agrupamiento de los argumentos de los estudiantes en función de los términos bases de mayor frecuencia revela básicamente una secuencia argumentativa como la que se expone enseguida: Tarea escolar PLT hacer PLT tiempo PLT comprensión PLT refuerzo PLT aprendizaje PLT sacar notas. En los argumentos que justifican la continuidad de las tareas escolares, a pesar de las deficiencias que se les critican, los estudiantes defienden estas actividades porque permiten efectuar acciones en el tiempo libre con miras a comprender, a reforzar y a aprender para, de esta manera, obtener notas. Según los datos recogidos, y a partir de lo identificado, se advierte que predomina una concepción distorsionada tanto de la tarea escolar como de las prácticas educativas.

Las representaciones de tarea escolar portan esencialmente valores pragmáticos positivos, intelectuales y epistémicos. En este orden de ideas, la valoración contenida en los discursos de los estudiantes que reclaman la continuidad de las tareas se orienta hacia lo útil que puede resultar este tipo de actividad escolar, más por los beneficios en términos de notas y de logros cuantitativos que por los aprendizajes y la transformación positiva de los sujetos. Entonces la tarea se asocia a conocimiento, a saber, lo que genera interés y motivación, pero especialmente porque llevará a alcanzar propósitos cuantitativos, recompensas y beneficios que deforman el sentido de la educación.

\section{Referencias}

Castillo, M., Santiago, W. y Ruiz, J. (2014). La representación del significado léxico y la movilización de valores sociales: la tarea escolar. Folios, 40, 105-124.

Devin, F. (2009). Approches dialogiques et énonciatives de l'interculturel : pour une didactique des langues et de l'identité mouvante des sujets. Synergies Roumanie, 4, 165-178. 
Galatanu, O. (2000). Signification, sens et construction discursive de soi et du monde. En J.M. Barbier y O. Galatanu (eds.), Signification, sens, formation (pp. 25-43). Paris : PUF.

Galatanu, O. (2002). Le concept de modalité : Les valeurs dans la langue et dans le discours. En O. Galatanu y F. Le Roy (eds.), Les valeurs, séminaire "le lien social" (pp. 17-32). Nantes : Maison des Sciences de l'Homme.

Galatanu, O. (2003a). La sémantique des possibles argumentatifs et ses enjeux pour l'analyse du discours. En I. Iñarrea Las Heras y M. J. Salinero Cascantel (coord.). El texto como encrucijada: estudios franceses y francófonos (pp. 213-226).

Galatanu, O. (2003b). La construction discursive des valeurs. En J.M. Barbier (eds.), Valeurs et activités professionnelles (pp. 87-114.). Séminaire du Centre de Recherche sur la Formation du CNAM. Paris: L'Harmattan.

Galatanu, O. (2005). Analyse du discours. Diversité Ville, école, intégration, 140, 55- 61. Recuperado en http:// www.cndp.fr/archivage/valid/70174/70174-1077813726.pdf.
Galatanu, O. (2007). Sémantique des possibles argumentatifs et axiologisation discursive. En D. Bouchard y J. Evrard (eds.), Représentation du sens linguistique 2 (pp. 313-325). Louvain-La-Neuve : Duculot De Boeck.

Galatanu, O. (2009). Les incidences sémantiques des déploiements argumentatifs dépendants du co-(n) texte de production du discours. Mémoires de la Société Néophilologique de Helsinki, LXXVIII, 391-404.

Putnam H. (1975). The meaning of "meaning". Philosophical papers, 2, 215-271.

Real Academia Española (RAE) (2003). Diccionario de la lengua española [Vigésima segunda edición. Edición electrónica Versión 1.0]. Madrid: Espasa Calpe.

Ruiz, J., Santiago, W. y Castillo, M. (2018). La tarea escolar: Concepciones de los estudiantes. Folios, 48, 101-119.

Ruiz, J., Castillo, M. y Santiago, W. (2016). Aproximación la representación del significado léxico de la entidad léxica tarea escolar. Forma y Función, 29(1), 151-177.

Ruiz, J., Castillo, M. y Santiago, W. (2015). Las tareas escolares: representaciones de los docentes de lenguas. Folios, 42, 105-126. 